\title{
Evaluation of dynamic microbial communities in a styrene-degrading biotrickling filter using 16S rDNA tag pyrosequencing and denaturing gradient gel electrophoresis
}

\author{
Kevin Portune, Maria Carmen Pérez, Francisco Javier Alvarez-Hornos, Carmen \\ Gabaldón
}

Accurately characterizing microbial communities within bioreactors undergoing dynamic operating conditions is an essential first step towards understanding the relationship between microbial community structure and bioreactor performance. A detailed assessment of the changes in microbial populations within a styrene-degrading biotrickling filter was carried out using samples collected at multiple time points ranging from 21 to 155 days of biotrickling filter operation. Examination of microbial populations was conducted by $16 \mathrm{~S}$ rDNA tag pyrosequencing and denaturing gradient gel electrophoresis (DGGE). Validation of pyrosequencing results was performed by quantitative polymerase chain reaction (qPCR) in order to examine the relative changes in percentages of selected taxonomic groups. Pyrosequencing results revealed a predominance of bacteria assigned to the phylum Proteobacteria for all sampling time points in the bioreactor. Relative fluctuations in percentages of total bacteria sequences assigned to selected taxonomic groups detected by pyrosequencing during biotrickling filter operation were confirmed by qPCR. Pyrosequencing revealed substantial changes in the community structure between sampling time points, with observed differences in microbial diversity indices and operational taxonomic units (OTUs) among certain samples. DGGE further revealed shifts in the dominant microbial species during changes in biotrickling filter operational parameters. The application of several different molecular tools to examine changes within microbial populations from bioreactors allows a more detailed view of the community structure as compared to using only one molecular method. This study highlights both the complementary as well as contrasting information that can be obtained in characterizing microbial populations using multiple molecular methods. 